\title{
Assessment of Hepatitis B vaccination status and needle stick injury awareness among doctors of a tertiary care medical college hospital
}

\author{
S. Rajesh*, R. Vidhya Rani, T. Sundararajan and Ismath Jahan \\ Department of Microbiology, Govt. Mohan Kumaramangalam Medical College, Salem, India
}

*Corresponding author

\section{Keywords}

Hepatitis B, Vaccination Status, Exposure-Prone Procedure (EPP)

\section{Article Info}

Accepted:

28 July 2016

Available Online:

10 August 2016

\section{A B S T R A C T}

Doctors are more prone to get needle stick injury while giving $\mathrm{i} / \mathrm{v}$ injection and during suturing process. HIV, Hepatitis B and Hepatitis C are three important fatal infections transmitted by needle stick injury. The transmission rate of Hepatitis $\mathrm{B}$ infection is nearly about $30 \%$ whereas transmission of HIV is only $0.3 \%$ and Hepatitis C only $1.8 \%$ and Hepatitis B being fatal can very well be prevented by proper vaccination and prophylaxis. After infection with $\mathrm{HBV}, 10 \%$ of the patients develop chronic hepatitis and about 15\%-25\% develop cirrhosis. Half of these individuals later develop hepatic decompensation or hepatocellular carcinoma. The study was conducted in Medical College Hospital, from May 2014 to July 2014. It was a descriptive, cross-sectional questionnaire based study. Consent was taken before the distribution of the questionnaire. All doctors were included in the study i.e. Obstetrician/Gynaecologists, Superspecialists, Anaesthetists, Paediatricians, Orthopaedician, Surgeons and Physicians. Forms were distributed among 345 doctors. Out of 345 distributed forms, 208 were returned with a response rate of $60.28 \%$.A total of 208 doctors, (age range 30-58 years) responded to the questionnaire. Amongst them $74 \%$ have been vaccinated and $26 \%$ have not completed the required course of vaccination. The most commonly cited reasons for non-vaccination found in our study were forgetting dosage schedule(43.9\%), and never thought of due to busy work pattern. It is seen that most of the young doctors have been vaccinated against $\mathrm{HBV}$ and the reason cited by them was as per government norms they had to get vaccinated during their under graduate and post graduate admissions. OG consultants (100\%), superspecialists $(90 \%)$ and anaesthetists $(90 \%)$ were more likely to have received completed vaccination, $85 \%$ of the paediatricians were completely vaccinated and the complete vaccination status was about $80 \%$ among surgeons, orthopaedicians, physicians. Needle stick injury was reported in $44.8 \%$ of the doctors. Needle stick injury reporting was fairly adequate and $88 \%$ of doctors were aware of post exposure prophylaxis. Post vaccination antibody testing for anti HBs levels were known only to few doctors(13\%). It is mandatory to identify low or non responders(anti $\mathrm{Hbs}<10 \mathrm{mlU} / \mathrm{ml}$ ) since they are not protected and post exposure prophylaxis should be initiated promptly after needle stick injuries and other exposures.

\section{Introduction}

Hepatitis $B$ is the major infectious occupational health hazard for the healthcare workers.
Health care workers are at higher risk of getting needle stick injury. Among health care workers, doctors are more prone to get 
needle stick injury while giving i/v injection and during suturing process. HIV, Hepatitis B and Hepatitis C are three important fatal infections transmitted by needle stick injury. The transmission rate of Hepatitis B infection is nearly about $30 \%$ whereas transmission of HIV is only $0.3 \%$ and Hepatitis C only $1.8 \%$ and Hepatitis B being fatal can very well be prevented by proper vaccination and prophylaxis (Pruss et al.,., 2005).

After infection with HBV, $10 \%$ of the patients develop chronic hepatitis and about $15 \%-25 \%$ develop cirrhosis. Half of these individuals later develop hepatic decompensation or hepatocellular carcinoma.

Health care workers have an increased risk of acquiring hepatitis B as they attend infected patients and thus are exposed to blood and body secretions of patients. According to Centre for Disease Control, 500-600 Health care workers (WCHs) are hospitalized annually due to exposure to blood products, of which more than 200 develop chronic hepatitis. The transmission of $\mathrm{HBV}$ requires direct, intimate contact with or parenteral inoculation of blood and blood products, semen or tissues.

"High Transmitter Risk"-including surgeons, operating room nurses, intensive care staff, interventional physicians, phlebotomists and emergency department staff and "Low Transmitter Risk" which includes all the remaining staff. Most (58\%) report exposure during a procedure and some $(37 \%)$ after the procedure. The risk of transmission of an infection from HCWs to patient is also great. There has been an increase in the number of such reports. Since 1972, 50 outbreaks have been reported in which a total of 48 infected HCWs, (39 surgeons) transmitted the infection to approximately 500 people .(Puro et al., 2003). In India, Hepatitis B surface Antigen prevalence among the general population ranges from 2 to $8 \%$, where as among healthcare workers seroprevalence is two to four times higher than that of the general population (Varsha Singhal et al., 2009). Studies have reported that about $10 \%$ of the health care workers (HCWs) in India have acquired HBV infection.The risk for developing serologic evidence of $\mathrm{HBV}$ infection was $37 \%$ to $62 \%$ If the blood was positive for both HBsAg and hepatitis B e antigen the risk for developing Hepatitis B infection was 37 to $62 \%$, and $23 \%$ to $37 \%$ if the blood was positive for HBsAg and negative for hepatitis $\mathrm{B}$ e antigen. HCWs exposed to needle stick injuries, blood containing $\mathrm{HBV}$, or both are at risk for developing clinical hepatitis. (Harsha Kumar et al., 2015). Low vaccination rates among HCWs have been reported in South Africa, and India. (Sukriti et al., 2008). Post vaccination antibody testing is essential to know the adequate immunological priming has been achieved and to identify non responders. (Rao et al., 2008). No study has so far been conducted in western Tamilnadu. So, there was a need to establish the vaccination status of health care workers of the biggest tertiary care hospital, situated in Salem, Western Tamilnadu. Hence this study was carried out to assess the hepatitis $B$ vaccination status of doctors working in Medical College Hospital, Salem, India.

\section{Materials and Methods}

The study was conducted in Medical College and Hospital from May 2014 to July 2014. It was a descriptive, crosssectional questionnaire based study. Consent was taken before the distribution of the questionnaire. All doctors were included in the study i.e. Obstetrician/Gynaecologists, Superspecialists, Anaesthetists, Paediatri- 
cians, Orthopaedicians, Surgeons and Physicians. Forms were distributed among 345 doctors. A structured questionnaire was made and distributed among health care workers and medical students. The questionnaire contained socio-demographics of doctors, screening status for Hepatitis B, Hepatitis B vaccination status, knowledge and attitude regarding Hepatitis $\mathrm{B}$ infection and knowledge of safe practices recommended for prevention of infection while examining patients, needle stick injury and post exposure prophylaxis. Complete vaccination refers to who received three doses of hepatitis $B$ vaccination at 0,1 and 6 month, Partially vaccination refers to who received 1 or 2 dose of hepatitis $B$ vaccination and not immunized means, who had not received any dose of vaccine.

\section{Results and Discussion}

Doctors have a greater probability of acquiring hepatitis $\mathrm{B}$ infection by virtue of their profession being occupationally exposed. The need for vaccination against HBV infection should be considered a priority as HBV vaccine is highly effective with 95\% seroconversion rates. Doctors are vital in controlling the epidemic of Hepatitis $B$ by effective vaccination and taking protective measures while dealing with patients.

Out of 345 distributed forms, 208 were returned with a response rate of $60.28 \%$.A total of 208 doctors, (age range 30-58 years) responded to the questionnaire.

The present study showed complete vaccination status among $74 \%$ of doctors and partially vaccinated status among $26 \%$ of doctors(Figure 1). Singal V et al., (2011) in their study have shown that out of $446 \mathrm{HCWs}, 224$ (50\%) HCWs were fully vaccinated and $28(6 \%)$ had received incomplete vaccination.

Figure.1 Vaccination status among doctors

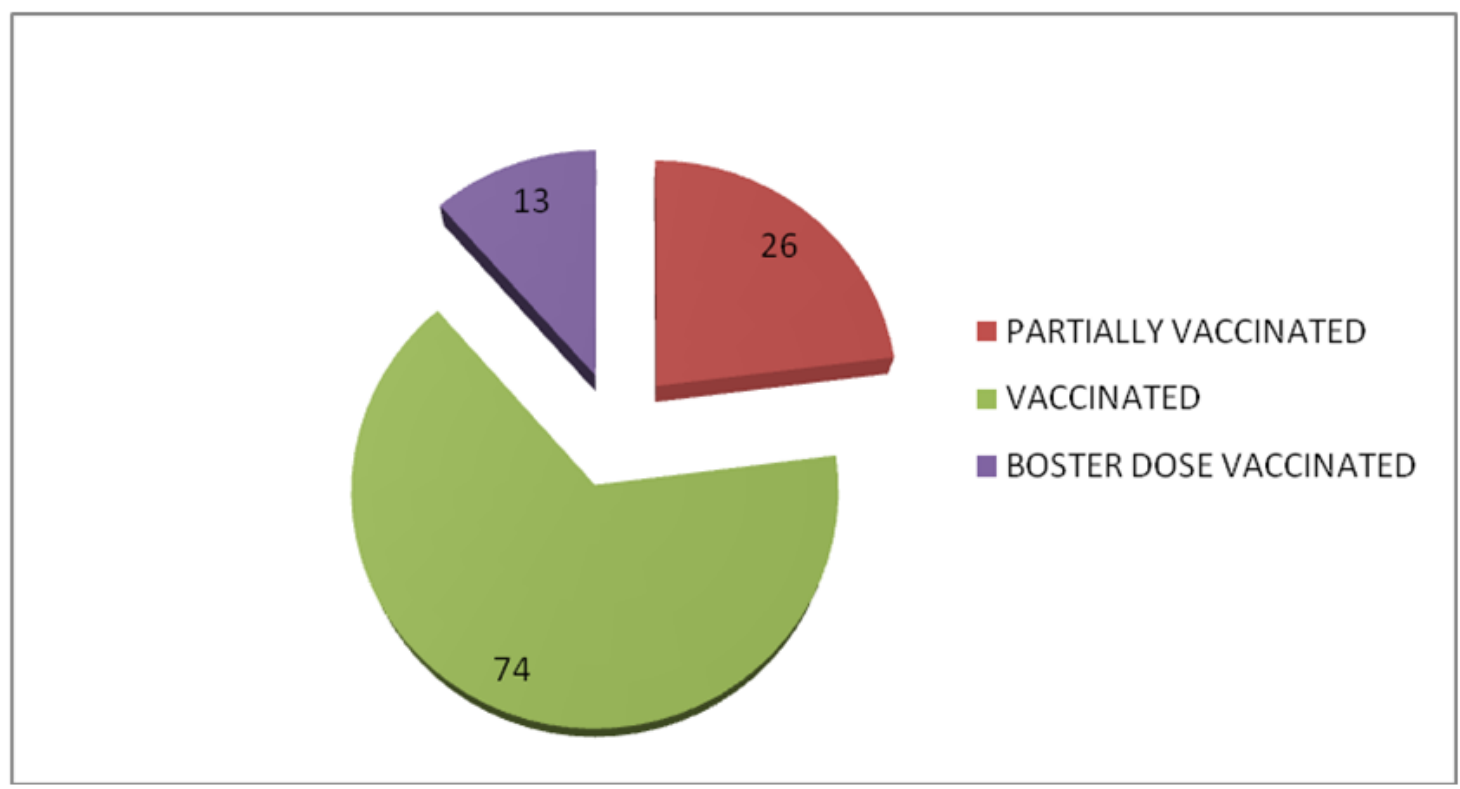


Figure.2 Percentage of Vaccination status among different Specialty of Doctors

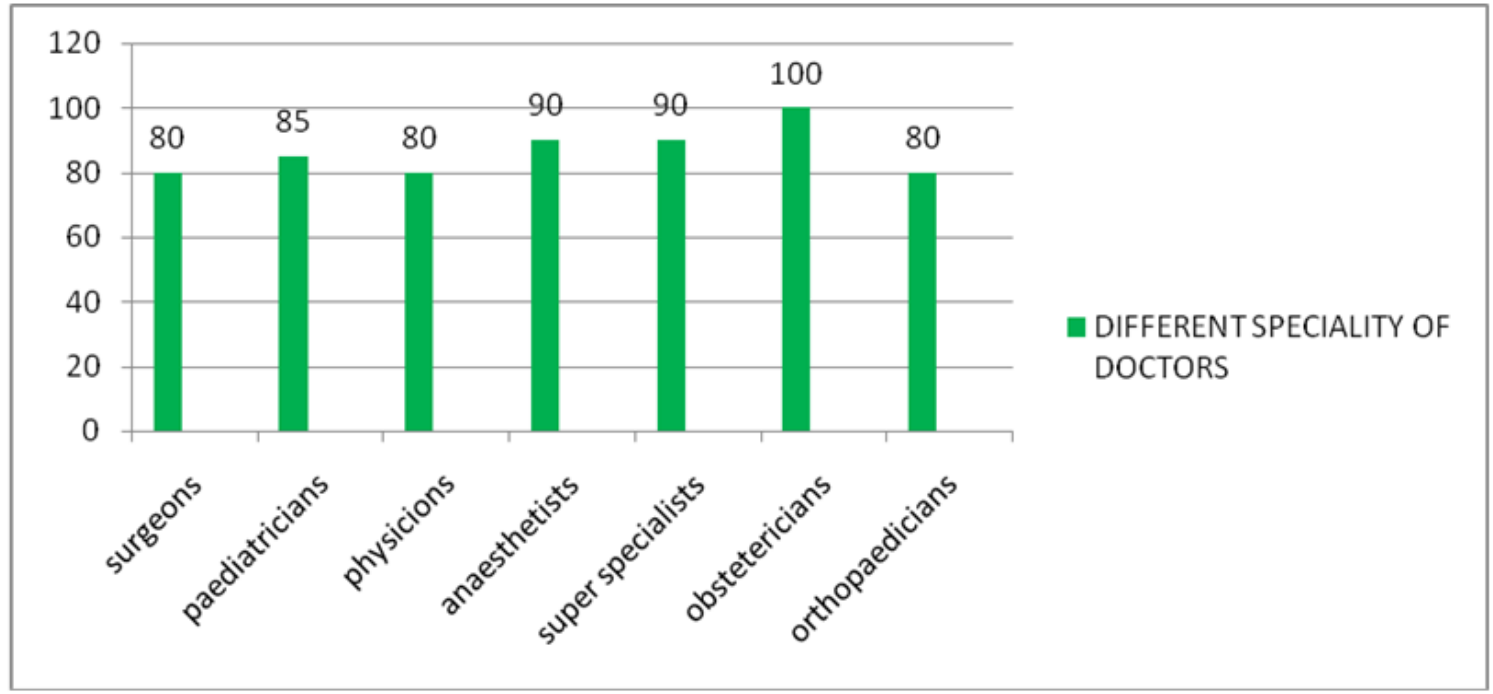

Figure.3 Percentage of Vaccination status among senior and junior and male/female Doctors

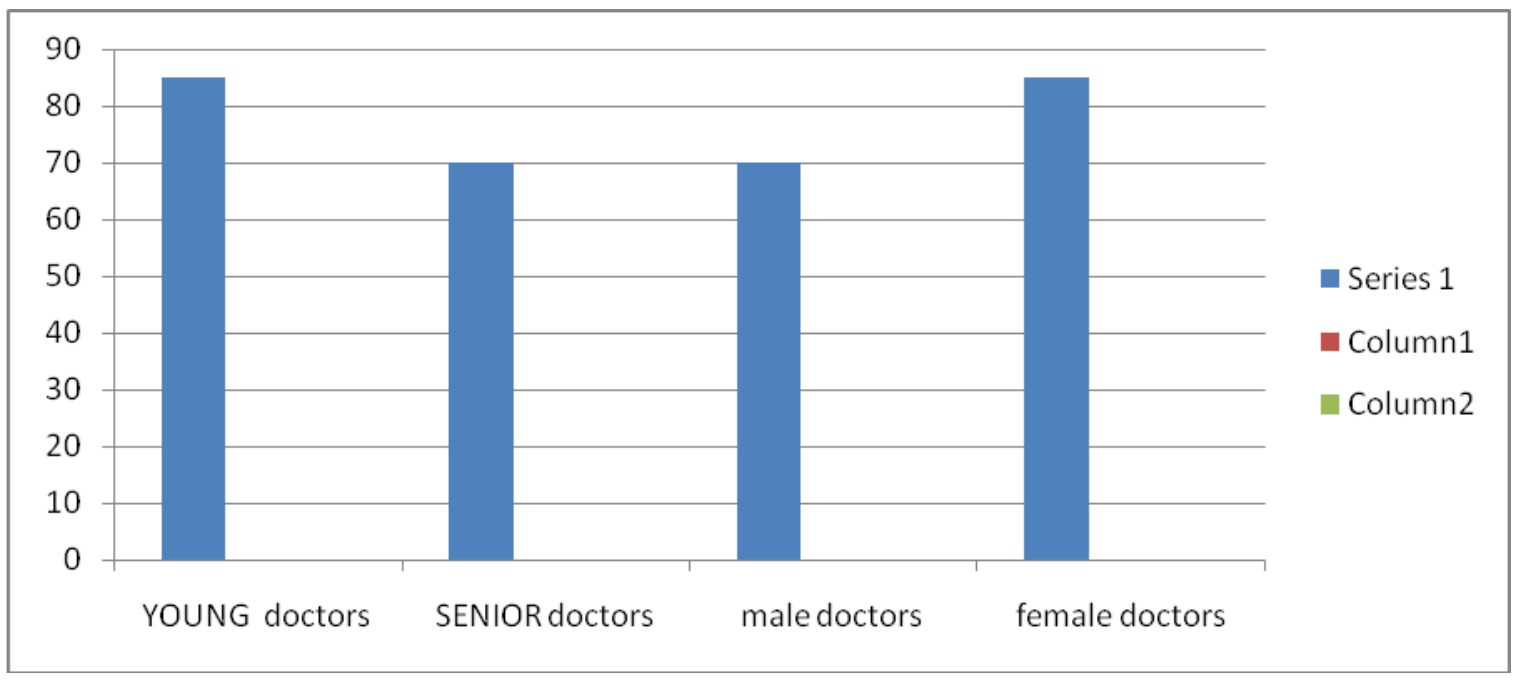

The most commonly cited reasons for nonvaccination found in our study were forgetting dosage schedule(43.9\%), and never thought of due to busy work pattern and hectic life style (56.1\%). Gaudour et al., (2011) had documented none specified cause as the reason for non vaccination.

In this study the complete vaccination status among the various speciality doctors were OG consultants $(100 \%)$, super-specialists (90\%) anaesthetists (90\%), Paediatricians
(85\%), surgeons (80\%), Orthopaedicians $(80 \%)$ and Physcians $(80 \%)$ more likely to have received completed vaccination (Figure. 2).

In our study Needle stick injury was reported in $44.8 \%$ of the doctors. . About $88 \%$ of doctors were aware of post exposure prophylaxis of needle stick injuries the reporting of needle stick injuries were fairly adequate. Rakesh et al., 2010 in their study had documented that $36 \%$ health care 
workers who had a history of needle stick injuries, about 92\% never reported the incident to a doctor to get post-exposure treatment because they were not aware of the importance of post-exposure prophylaxis. Needle stick injuries pose a greater risk than splashes and those from hollow-bore needles.

In this study very few (13\%) were knowing their post vaccination antibody titre of anti HBs after completing vaccination. TV Rao et al., had shown in their study that $10.5 \%$ ) of the successfully vaccinated health care workers who were low or non-responders $(<10 \mathrm{mlU} / \mathrm{ml})$. The Tamilnadu Government had conducted needle stick injury prevention awareness programs through Tamilnadu health systems project in all government medical college hospitals. Many of doctors had acquired good knowledge on management of needle stick injury, its prevention and post exposure prophylaxis.

Only young doctors who has less than 10 years of government service have completed their vaccination about $85 \%$ and Only $70 \%$ Of senior doctors had completed the course of Hepatitis B vaccination but Many of senior doctors were only partially vaccinated due to busy work pattern. But Rabia arshed usmani et al.,(2010) shown in their study at 2010 as $83 \%$ of senior doctors completely vaccinated and $70 \%$ junior doctors less than 5 to 10 years service had vaccinated completely. It is seen that most of the young doctors have been vaccinated against HBV and the reason cited by them was as per government norms they had to get vaccinated during their under graduate and post graduate admissions. Many of the senior doctors have not completed their vaccination because no completion and no such awareness before 15 years. Some of the senior doctors got complete vaccination only after their first episode of needle stick injury.

In conclusion, the most commonly cited reasons for non-vaccination and in complete vaccination found in our study were forgetting dosage schedule(43.9\%), and never thought of due to busy work pattern and hectic life style of doctors. The study shows that though large numbers of doctors were completely vaccinated against Hepatitis B only few doctors were aware of post vaccination antibody testing for antiHBs levels. There is a need to ensure that every doctor is completely vaccinated against Hepatitis B before he/she enters professional practice and the importance of post vaccination antibody testing to identify non responders known only to few doctors $(13 \%)$. It is essential to identify low or non responders (anti $\mathrm{Hbs}<10 \mathrm{mlU} / \mathrm{ml}$ ) since they are not protected and post exposure prophylaxis should be initiated promptly after needle stick injuries and other exposures. Our study shows the need for a more aggressive approach to doctors for $100 \%$ complete vaccination for their own benefit. $100 \%$ awareness can be created by repeated orientation program and conducting free vaccination programs.

\section{References}

Harsha Kumar,HN Rahul P. Nambiar, Sarbjit Mohapatra,Aditi Khanna, R. Praveen,D. Sai Bhawana 2015. A Cross-sectional Study on Hepatitis B Vaccination Status and Post-exposure Prophylaxis Practices Among Health Care Workers in Teaching Hospitals of Mangalore Annals of Global Health. Volume 81, Issue 5, p587-732

Mohammed O. H. Gadour and Abdulazim M.O. AbdullahJune 2011 Knowledge of HBV Risks and Hepatitis B 
Vaccination Status Among Health Care Workers at Khartoum and Omdurman Teaching Hospitals of Khartoum State in Sudan.. sudan journal of medical sciences. 3.volume 6, Number 2 (), 63-68

Prüss-Üstün A， Rapiti E, Hutin Y (2005) Estimation of the global burden of disease from sharps injuries to health-care workers. Am J Ind Med 48: $482-490$.

Puro $\mathrm{V}^{1}$, Scognamiglio P, Ippolito G.2003. $\mathrm{HIV}, \mathrm{HBV}$, or HCV transmission from infected health care workers to patientsMed Lav.3NovDec;94(6):556-68.

Rabia arshed usmanimuhammad saleem wazir, huda sarwer, 2010. Assessment of hepatitis $b$ vaccination status in doctors in Lahore,.j ayub med collabbottabad 2010 apr-jun 22(2):369.

Rakesh Shah, H.K. Mehta, Manish Fancy, Sunil Nayak, Bhavesh N. Donga,2010.Knowledge And Awareness Regarding Needle Stick Injuries Among Helath Care Workers
In Tertiary Care Hospital In Ahmedabad , Gujarat. National Journal of Community Medicine Vol. Issue 2

Rao TV, IJ Suseela, KA Sathiavathy.2008 Estimation of antibodies to HBsAg in vaccinated health care workers. Indian journal of Medical Microbiology. 26 (1): 93-94

Singhal V, Bora D, Singh S (2011) Prevalence of Hepatitis B Virus Infection in Healthcare Workers of a Tertiary Care Centre in India and Their Vaccination Status. J Vaccines Vaccin 2:118.

Sukriti, Pati, N.T., Sethi, A. et al.,.2008 Low levels of awareness, vaccine coverage, and the need for boosters among healthcare workers in tertiary care hospitals in India. J Gastroenterol Hepatol. ;23: 1710-1715

Varsha Singhal, Dhrubajyoti Bora, and Sarman Singh.2009. Hepatitis B in Health Care Workers: Indian Scenario J Lab Physicians. Jul-Dec; 1(2): 4148.

\section{How to cite this article:}

Rajesh, S., R. Vidhya Rani, T. Sundararajan, and Ismath Jahan 2016. Assessment of Hepatitis $\mathrm{B}$ vaccination status and needle stick injury awareness among doctors of a tertiary care medical college hospital. Int.J.Curr.Microbiol.App.Sci. 5(8): 895-900. doi: http://dx.doi.org/10.20546/ijcmas.2016.508.101 